\title{
Estimation de la digestibilité de l'herbe ingérée au pâturage à partir de l'azote fécal et de quelques autres paramètres fécaux
}

\author{
M. CHENOST \\ avec la collaboration technique de J.M. BOISSAU \\ I.N.R.A., Laboratoire des Aliments, C.R.Z.V. de Theix \\ F 63122 Ceyrat
}

\begin{abstract}
Résumé
L'objet de cette étude est de proposer des équations permettant de prédire la digestibilité de l'herbe pâturée à partir de quelques critères fécaux et de discuter les conditions d'utilisation de ces équations dans la pratique.

On a utilisé pour cela les résultats de mesure de la digestibilité, effectuée sur moutons, de 1230 échantillons (fourrages verts) des 10 principales espèces prairiales exploitées en France et de deux prairies naturelles.

On a calculé les équations de régression liant la digestibilité de la matière organique (dMO) des fourrages verts aux teneurs en matières azotées et en cellulose brute (MATF et $\mathrm{CBF}$, exprimées en p. 100 de la matière organique) dosées sur les fèces correspondantes. La digestibilité des fourrages est comprise entre 55 et 80 p. 100 et la teneur en MATF entre 8 et $30 \mathrm{p} .100$.
\end{abstract}

Les liaisons entre dMO et MATF sont étroites et CBF, ajoutée à MATF, n'améliore pas significativement la précision de la prévision de dMO.

Les équations et les courbes correspondantes ( $26 \mathrm{du} 2^{\circ}$ degré, $5 \mathrm{du} 1^{\text {er }}$ degré) sont donc présentées en fonction de MATF seule. Ces 31 équations ont dû être établies espèce par espèce et cycle par cycle. En effet, à teneur en MATF donnée et suivant les cycles successifs :

- la digestibilité diminue, quelle que soit l'espèce botanique;

- les différences de digestibilité entre espèces botaniques augmentent;

- le classement des différentes espèces suivant leur digestibilité n'est pas le même.

Les coefficients de corrélation sont compris entre 0,887 et 0,958 pour les premiers cycles et entre 0,735 et 0,910 pour les différentes repousses. La précision de la prévision (Syx) de dMO est en moyenne de 2,2 à 2,5 points ( 3 valeurs sur 31 dépassent 3 points). Elle est voisine et même parfois meilleure que celle obtenue à partir de la teneur en matières azotées et en cellulose brute du fourrage offert (MATP et CBP respectivement).

Sur un nombre d'échantillons plus restreint (29 ray-grass d'Italie, 17 dactyles) on a vérifié que la précision de la prévision pouvait être encore améliorée [Syx $=1,28$ et 1,67 points respectivement pour le ray-grass d'Italie et le dactyle) en ajoutant à MATF un ensemble de critères fécaux chimiques : $\mathrm{CBF}$, matière sèche soluble dans l'eau (MSEF), constituants pariétaux (NDF) et lignocellulose (ADF) de Van SoEST], et physiques : densité des fèces (DENS F). On peut même apprécier la quantité de matière organique digestible ingérée avec une bonne précision $\left(1,28\right.$ à $\left.2,33 \mathrm{~g} / \mathrm{kg} \mathrm{P}^{0,75}\right)$ en ajoutant MATP et CBP à l'ensemble de ces critères fécaux. 
La discussion passe en revue les conditions d'application de ces équations dans la pratique : utilisation pour prévoir la digestibilité de l'herbe pâturée par des bovins, avec ou sans complémentation au pâturage et pour des niveaux d'alimentation inférieurs à l'ad libitum.

En conclusion, ces équations, établies pour des prairies monospécifiques, peuvent être utilisées dans la pratique sous certaines conditions décrites dans le texte. Elles permettent, à partir d'un échantillon de fèces représentatif d'une période de pâturage donnée, de prévoir et de classer les digestibilités de l'herbe pâturée par des moutons et des bovins.

Le problème des prairies naturelles plurispécifiques reste entier. On doit alors avoir recours soit à des mesures de digestibilité sur moutons conduites parallèlement au pâturage avec la même herbe fauchée, soit à des mesures de laboratoire (cellulase, sachets de nylon, DIV) permettant la prévision de dMO à partir d'un échantillon d'herbe représentatif de l'herbe pâturée (fauche, bol œsophagien).

Mots clés : liaisons digestibilité herbe, caractéristiques fécales.

\section{Introduction}

La connaissance des quantités d'herbe ingérées par l'animal au pâturage est intéressante pour préciser la complémentation à apporter et pour micux expliquer les résultats zootechniques enregistrés.

Plusieurs méthodes ont été proposées pour les prévoir. Ces méthodes sont soit indirectes (fauche avant et après passage des animaux sur la parcelle), soit directes : estimation de la quantité de fèces émises et de la digestibilité de l'herbe pâturée ; utilisation des performances réalisées par les animaux, les besoins étant connus; enregistrement du comportement alimentaire de l'animal : produit du nombre de préhension par le poids moyen de la bouchée d'herbe récoltée à chaque préhension.

C'est l'estimation de la quantité de fèces émises et de la digestibilité de l'herbe pâturée qui a fait l'objet du plus grand nombre de travaux (revues de StreEter, 1969 ; Langlands, 1975 ; Meijs, 1981 ; Le Du \& Penning, 1982 et de HolecheK et al., 1982).

La quantité ingérée (I) est égale à $\frac{\mathrm{F}}{100-\mathrm{D}}$ où $\mathrm{D}$ est la digestibilité de l'herbe

pâturée et F la quantité de fèces émises. Celle-ci peut être mesurée par collecte totale (animaux porteurs de sacs de collecte) ou estimée par l'administration d'une quantité connue d'un marqueur externe $\left(\mathrm{Cr}_{2} \mathrm{O}_{3} \ldots\right)$ non digéré par l'animal et dont on dose la concentration dans les fèces.

La digestibilité (D) de l'herbe pâturée peut être estimée à partir de la digestibilité des bols alimentaires prélevés lors de l'ingestion sur des animaux munis d'une fistule de l'œsophage. La digestibilité des bols est prévue à partir à leur composition chimique, ou mieux, de leur digestibilité in vitro (DIV), (TILley \& Terry, 1963) ou de leur digestibilité par la pepsine cellulase (Aufrere, 1982).

La digestibilité de l'herbe pâturée par les animaux peut également être estimée à partir de la composition chimique des fèces (teneur en azote, en chromogènes, en groupement méthoxyl, en silice, etc.). Parmi ces caractéristiques, c'est la teneur en 
azote fécal qui, depuis les travaux de Lancaster (1949) et Raymond, Kemp \& Harris (1954), a été le plus utilisée.

Grâce aux études de Demarouilly et al. (1978), nous disposons au Laboratoire de plus de 1250 échantillons de fourrages verts dont on avait mesuré la digestibilité chez le mouton. Les teneurs en cendres, en matières azotées totales et en cellulose brute, avaient été dosées sur les échantillons de fourrages et des fèces correspondantes.

L'objet de cette étude a donc été de 1) calculer les relations liant la digestibilité de la matière organique des diverses espèces prairiales pures et deux prairies permanentes à la teneur en matières azotées et en cellulose brute des fèces des animaux ayant servi à mesurer leur digestibilité ; 2) voir sur certains de ces échantillons, choisis pour couvrir une gamme de digestibilité assez large, si le fait d'ajouter d'autres caractéristiques (de l'herbe elle-même ou des fèces) permettrait d'améliorer la prévision de la digestibilité, ou même de prévoir directement la quantité de matière organique digestible ingérée ; 3) étudier les conditions d'application de ces équations dans la pratique.

\section{Matériel et méthodes}

\section{A. Choix des échantillons}

Au total, nous avons utilisé les résultats enregistrés sur 1230 échantillons dont le nombre par espèce botanique et par cycle est reporté dans le tableau 1.

La digestibilité des fourrages a été mesurée sur des lots de 6 moutons maintenus en cages à bilan. Les moutons recevaient le fourrage frais, fauché le matin, en 2 repas par jour et à volonté (pourcentage de refus tolérés compris entre 10 et $15 \mathrm{p} .100$ ). Les mesures étant effectuées en continu pour chaque cycle, une période de mesure ( 6 jours de collecte) donnée servait de période de mise en régime pour la semaine suivante.

Nous avons considéré les fourrages espèce par espèce et, à l'intérieur d'une espèce donnée, cycle par cycle. En effet, les relations entre la digestibilité et la teneur en matières azotées des fèces diffèrent, entre autres, selon l'espèce botanique (LANGLANDS, 1962, Langlands, CorbetT \& MC Donald, 1963) et le cycle de repousse (GreenHALGH \& CORBETT, 1960 ; Minson \& KEMP, 1961).

Nous n'avons pas retenu les fourrages exploités lors de l'année de semis car leur nombre par cycle, à l'exception du ray-grass d'Italie annuel, était trop faible pour calculer une équation de régression. Nous avons également éliminé 20 échantillons de repousses dont la teneur en matières azotées des fèces s'écartait trop, à digestibilité donnée, du reste de la population.

Nous avons enfin été amenés à regrouper certains cycles $\left(2^{\mathrm{e}}\right.$ et $3^{\mathrm{e}}$ ou $3^{\mathrm{e}}$ et $\left.4^{\mathrm{e}}\right)$ lorsque le nombre de mesures pour certains d'entre eux était insuffisant. Nous n'avons toutefois procédé à ce regroupement que dans la mesure où les graphiques reportant la digestibilité en fonction de la teneur en azote des fèces montraient que les points appartenaient à la même population. 


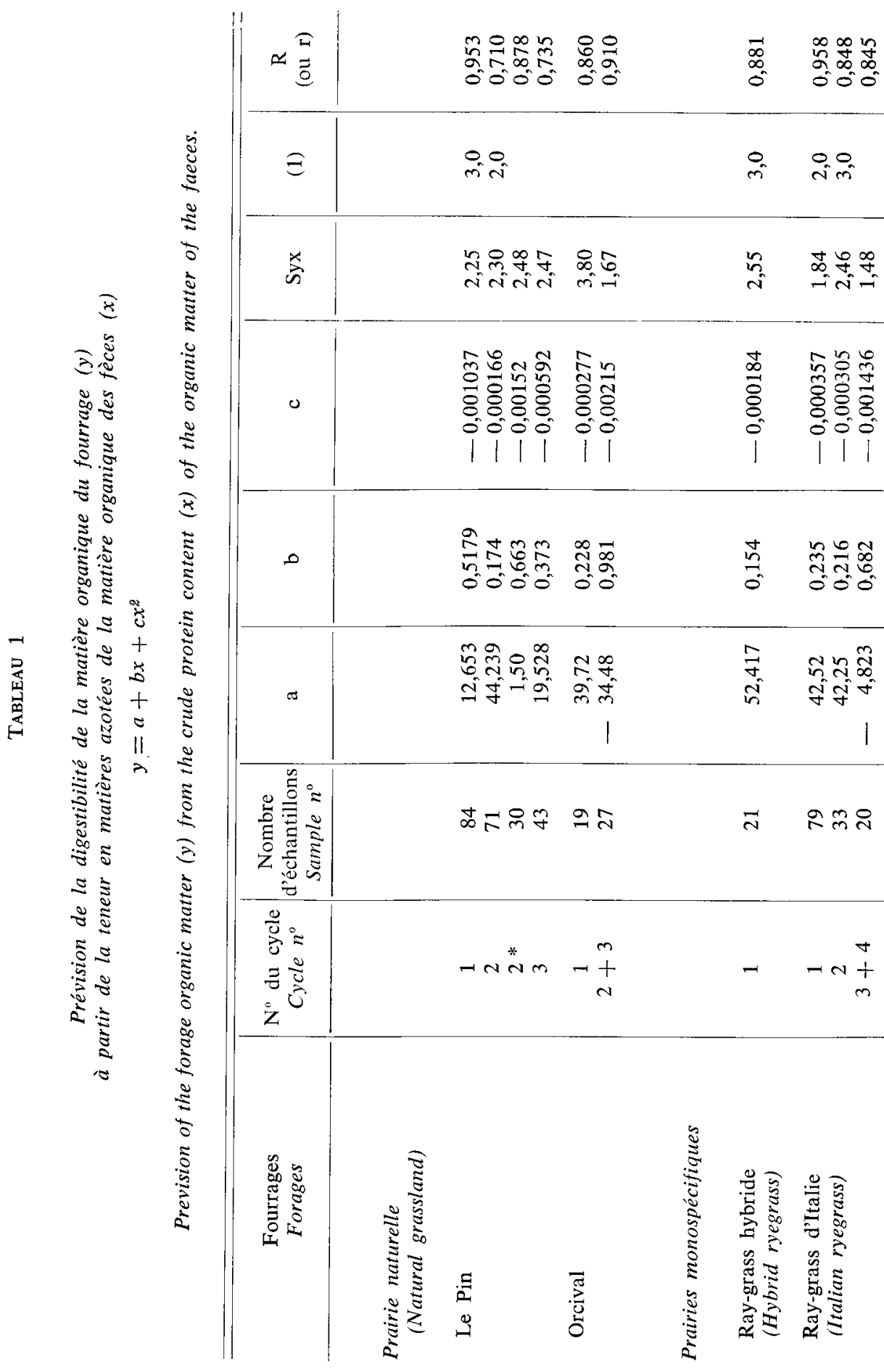


Les amplitudes de variation de la digestibilité de la matière organique (dMO) et de la teneur en matières azotées de la matière organique fécale (MATF ${ }_{\wp}$ ) sont très importantes pour les premiers cycles (de 55 à 85 et de 8 à 30 p. 100 respectivement). Elles diminuent avec le numéro du cycle : de 60 à 75 et de 10 à 27 p. 100 respectivement pour les $2^{\mathrm{c}}$ (ou $2^{\mathrm{e}}+3^{\mathrm{c}}$ cycles) et de $60-65$ à $70-75$ et de 15 à $26 \mathrm{p} .100$ respectivement pour les $3^{\mathrm{e}}$ (ou $3^{\mathrm{c}}$ et $4^{\mathrm{c}}$ cycles).

\section{B. Calculs}

Nous avons calculé les équations de régression liant la dMO (en points) du fourrage proposé à $\mathrm{MATF}_{\varnothing}$ et à la teneur en cellulose brute $\left(\mathrm{CBF}_{\emptyset}\right)$ (en p. 100) de la matière organique des fèces correspondantes.

Nous avons pour cela utilisé le modèle mathématique et le programme de calcul sur ordinateur décrit par SEEBEck (1973). Ce programme permet de rechercher la meilleure équation de régression entre la variable expliquée et les variables explicatives prises seules ou deux à deux, soit telles quelles, soit élevées au carré.

Nous avons voulu voir, sur un nombre plus restreint d'échantillons, si le fait de rajouter un certain nombre de caractéristiques fécales supplémentaires pouvait améliorer la prévision de la digestibilité et permettait également de prévoir la quantité de matière organique digestible ingérée (MODI). Nous avons choisi des échantillons de fèces (10 des premiers cycles et 7 des troisièmes cycles de dactyle, 19 des premiers cycles et 10 des deuxièmes cycles de ray-grass d'Italie) dont nous avions toutefois vérifié au préalable le bon état de conservation grâce à une nouvelle détermination des teneurs en matières azotées. Nous avons procédé aux analyses suivantes :

- constituants pariétaux (NDF) et lignocellulose (ADF) de vaN SoEsT (1967). Comme la teneur de ces constituants dans les fèces dépend de la fraction pariétale indigestible du fourrage (JARRIGE, 1965), laquelle détermine la digestibilité du fourrage, celle-ci est donc en liaison avec NDF et ADF des fèces. Il s'agit là d'une relation directe entre la dMO et les critères pariétaux des fèces, la liaison entre dMO et $\mathrm{MATF}_{\varnothing}$ n'étant, en fait, qu'une relation indirecte découlant de cette dernière ;

- matières solubles dans l'eau à $40^{\circ} \mathrm{C}$ (MSEF) (JARRIge 1965) : l'ensemble MATF + MSEF, donnant une estimation plus précise de la digestibilité de l'ensemble premiers cycles et repousses des graminées. Les fèces des premiers cycles de graminées contiennent en effet moins d'azote mais plus de MSE que les repousses ; MATF et MSEF doivent donc améliorer la prévision de la dMO de l'ensemble premiers cycles et repousses de graminées;

- densité des fèces (DENSF), appréciée par la hauteur occupée par $5 \mathrm{~g}$ de fèces broyées à la grille de $1 \mathrm{~mm}$ (et séchées à nouveau avant la mesure) déposées dans un cylindre de $1,8 \mathrm{~cm}$ de diamètre et soumise à un piston d'acier inoxydable d'un poids de $1 \mathrm{~kg}$ (JARrige, 1965). Cette densité caractérise la « fibrosité » des fèces qui augmente lorsque la plante vieillit alors que sa digestibilité diminue. Nous l'avons utilisée comme caractéristique physique des fèces.

Pour ces mêmes échantillons, nous avons également introduit deux variables explicatives supplémentaires : les teneurs en matières azotées et en cellulose brute

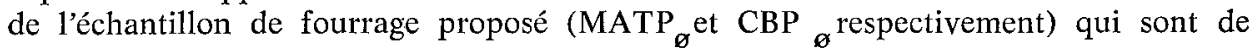


bons éléments de prédiction de la digestibilité, elle-même en liaison avec les quantités ingérées chez le ruminant.

Pour plus de simplicité et par souci d'homogénéité, nous n'utiliscrons que les abréviations MATF, CBF, MATP, CBP, étant entendu qu'elles sont exprimées en p. 100 de la matic̀re organique, et NDFF et DENSF exprimées en p. 100 de la matière sèche.

Nous avons calculé les équations de régression liant la digestibilité de la matière organique à MATF et $\mathrm{CBF}$ d'une part et aux diverses combinaisons MATF, CBF, NDFF, ADFF, MSEF, DENSF, MATP et CBP d'autre part.

\section{Possibilités d'utilisation de ces relations dans la pratique}

Les relations ont été établies sur moutons ne recevant que de l'herbe à volonté. Or dans la pratique nous avons le plus souvent à faire à des bovins consommant de l'herbe et du concentré. Le niveau d'alimentation pourra, en outre, ne pas correspondre à celui retenu lors des mesures de digestibilité.

Aussi, avons nous exploité les résultats de 3 autres essais, poursuivis par ailleurs au laboratoire, pour étudier l'influence du niveau d'alimentation et de la complémentation de l'herbe sur les liaisons dMO/MATF et voir dans quelle mesure les relations obtenues sur moutons pouvaient s'appliquer aux bovins.

\section{Résultats}

\section{A. Prévision de la digestibilité de la matière organique à partir des teneurs en matières azotées et en cellulose brute fécale}

Les équations permettant de prévoir la digestibilité de la matière organique avec la meilleure précision sont des équations du second degré où la variable explicative est MATF (tabl. 1). Ceci rejoint les observations de GreEnhalgh \& CorbetT (1960), Kennedy, Carter et Lancaster (1959) et Arnold \& Dudzinski (1963). Seules les équations obtenues pour le brome, les $2^{\text {cs }}$ cycles du ray-grass anglais, les $1^{\text {ers }}$ cycles du trèfle violet et les $2^{\text {es }}$ cycles de luzerne sont du premier degré.

CBF seule ou ajoutée à MATF a parfois amélioré, mais jamais de manière significative, la précision obtenue avec les teneurs en matières azotées fécales seules. Pour des raisons d'homogénéité dans la présentation des résultats nous n'avons donc pas reporté dans le tableau 1 les équations faisant intervenir la cellulose brute.

Ces équations $\mathrm{dMO}=\mathrm{f}(\mathrm{MATF})$ sont spécifiques de chaque espèce et de chaque cycle. En effet, nous n'avons pas pu effectuer les regroupements permettant de ne prendre en compte que l'effet moyen du cycle et/ou de l'espèce sur l'ordonnée à l'origine d'une équation de prévision générale.

Les coefficients de corrélation sont élevés : ils sont compris entre 0,957 et 0,860 pour les $1^{\text {ers }}$ cycles et entre 0,910 et 0,710 pour les repousses, sauf dans le cas du $2^{\mathrm{c}}$ cycle de dactyle $(0,942)$. 


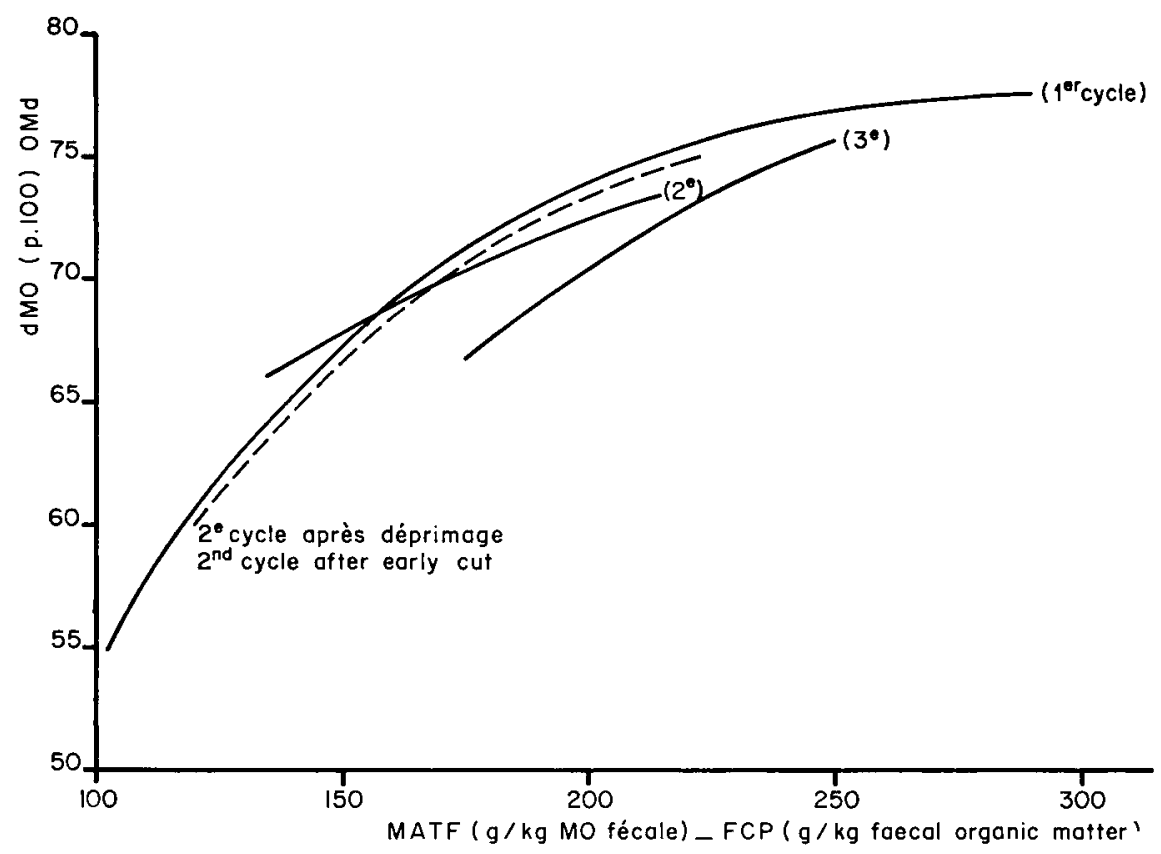

FIG. 1

Liaisons entre la digestibilité de la matière oganique (dMO) et la teneur en matières azotées totales de la matière organique fécale (MATF). Prairie naturelle Normande du Pin.

Relationships between organic matter digestibility (OMd) and total crude protein content of faecal organic matter.

La figure 1 donne un exemple des courbes obtenues pour les différents cycles d'une prairie donnée (prairie naturelle normande).

D'une manière générale, à teneur donnée en matières azotées fécales, la digestibilité diminue quand le numéro du cycle augmente ou, inversement, à digestibilité donnée, la teneur en matières azotées fécales augmente en même temps que le numéro du cycle, ce qui est en accord avec les résultats de Greenhalgh \& CorbetT (1960).

Ce phénomène est plus ou moins marqué suivant les espèces botaniques. Il l'est particulièrement pour la fétuque des prés, mais peut-être parce qu'elle comprenait une assez forte proportion de trèfle blanc aux $2^{\circ}, 3^{\text {e }}$ et $4^{\mathrm{e}}$ cycles.

Les courbes sont plus décalées les unes par rapport aux autres pour les $3^{\circ}$ et $4^{\mathrm{e}}$ cycles que pour les $1^{\mathrm{cr}}$ et $2^{\mathrm{e}}$ cycles; en outre leur position respective n'est pas la même d'un cycle à l'autre.

Nous donnons un exemple de ces courbes dans la figure 2 pour les $3^{\circ}$ et $4^{r}$ cycles. 


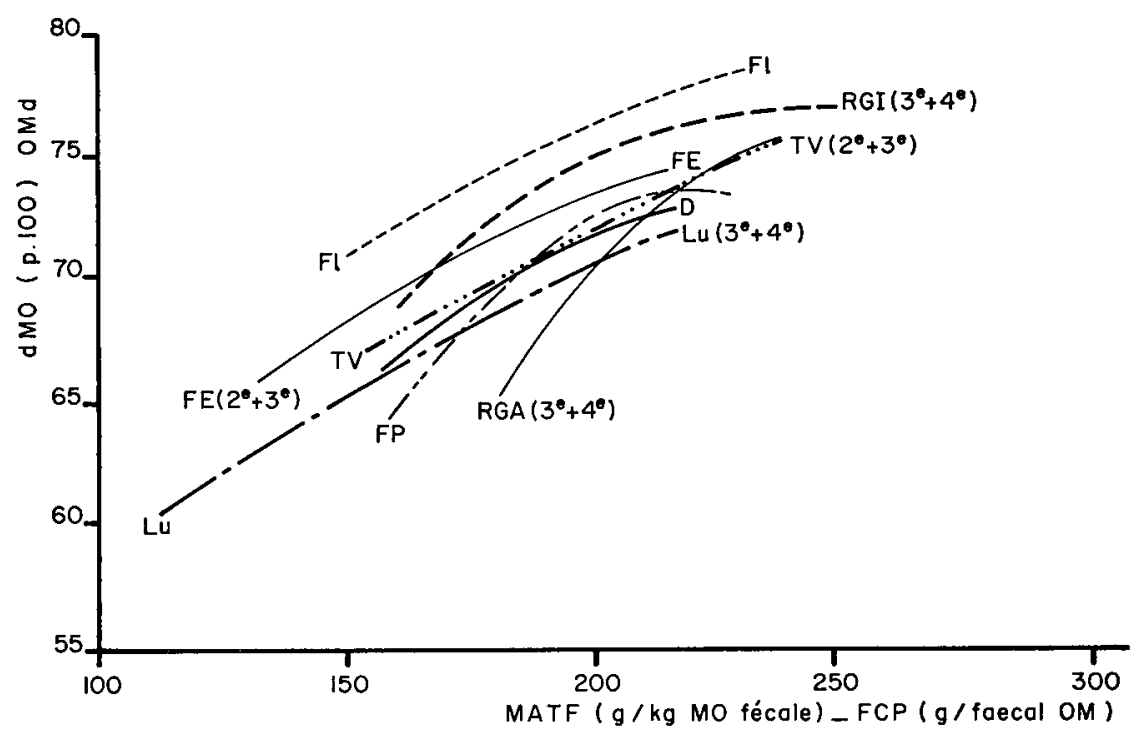

FIG. 2

Courbes de régression liant la digestibilité de la matière organique (dMO) des différents fourrages à la teneur en matières azotées des fèces (MATF) des moutons $\left(3^{e}\right.$ et $3^{e}+4^{e}$ cycles $)$.

Relationships between organic matter digestibility (OMd) and crude protein content of the sheep faeces (FCP) (3rd and $3 r d+4 t h$ cycles).

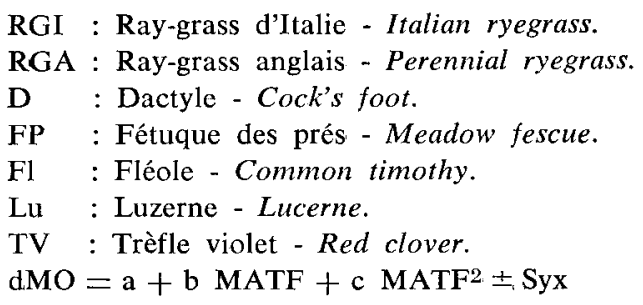

(Cf. tableau 1 pour les valeurs de a, b, c, Syx, R et $\mathrm{r}$ - See table 1 for $a, b, c$, Syx, $R$ and $r$ values).

Il est difficile de comparer la précision de la prévision (Syx) d'une espèce à l'autre et d'un cycle à l'autre car le nombre d'échantillons et les limites de variation de leur digestibilité ne sont pas les mêmes. La précision est généralement comprise entre 2,0 et 2,5 points, les extrêmes allant de 1,4 ( $2^{\circ}$ cycle de fétuque des prés) à 3,8 points ( $1^{\text {er }}$ cycle de la prairie naturelle d'Orcival). Les limites de variation de dMO sont plus faibles pour les repousses que pour les $1^{\text {ers }}$ cycles. C'est ainsi que la précision augmente (Syx diminue) avec le numéro du cycle pour l'ensemble des espèces, sauf pour la prairie naturelle du Pin, le ray-grass anglais et le brome alors que, parallèlement, les coefficients de corrélation diminuent. 
La prévision de dMO est moins bonne que celle observée par GreEnHalgh \& Corbett (1960), Greenhalgh, Corbett \& Mc Donald (1960) et Grelnhalgh, Reid \& Mc Donald (1966) et voisine de celle constatée par Minson \& Kemp (1961).

Les teneurs en cendres sont beaucoup plus variables dans les fèces que dans les fourrages correspondants. Nous avons pensé que l'introduction des cendres fécales pouvait améliorer la précision de la prévision de la digestibilité par rapport à MATF. Nous avons effectué les calculs pour les $1^{\text {er }}, 2^{\prime \prime}$ et l'ensemble $3^{\mathrm{c}}$ et $4^{\circ}$ cycles de la fétuque des prés; la précision n’est pas supérieure (tabl. 2).

Lorsque le nombre d'échantillons par espèce et par cycle était le même et rendait la comparaison possible, nous avons constaté que la précision de la prévision de dMO obtenue par ANDRIEU \& Weiss (1981) à partir de la teneur en matières azotées totales (en p. 100 de la matière organique) du fourrage proposé (MATP) était légèrement moins bonne que la nôtre (tabl. 1).

\section{TABLEAU 2}

Précision de la prévision de la digestibilité de la matière organique à partir de la teneur en matières azotées (MATF) seule ou avec la teneur en cendres des fèces (cas de la Fétuque des prés).

Accuracy of organic matter digestibility prediction from faecal crude protein content (FCP) alone or with faecal ash content (Meadow fescue).

\begin{tabular}{|c|c|c|c|c|c|}
\hline \multirow{2}{*}{$\begin{array}{l}\text { Numéro } \\
\text { du cycle } \\
\text { Cycle } n^{\circ}\end{array}$} & \multirow{2}{*}{$\begin{array}{c}\text { Nombre } \\
\text { d'ćchantillons } \\
\text { Sample number }\end{array}$} & \multicolumn{2}{|c|}{$\begin{array}{c}\text { MATF } \\
F C P\end{array}$} & \multicolumn{2}{|c|}{$\begin{array}{c}\text { MAT, cendres } \\
F C P \text {, ashes }\end{array}$} \\
\hline & & $\mathbf{R}$ & Syx & $\mathbf{R}$ & Syx \\
\hline 1 & 38 & 0,939 & 2,29 & 0,838 & 3,63 \\
\hline 2 & 26 & 0,899 & 1,38 & 0,876 & 1,52 \\
\hline $3+4$ & 26 & 0,775 & 2,19 & 0,508 & 3,02 \\
\hline
\end{tabular}

B. Prévision de la digestibilité de la matière organique

et de la matière organique digestible ingérée à partir de variables explicatives supplémentaires

(sur 19 échantillons du $1^{\text {er }}$ cycle et 10 échantillons du $2^{\circ}$ cycle de ray-grass d'Italie et sur 17 échantillons du $1^{\text {er }}$ et $3^{\circ}$ cycles de dactyle)

1. Prévision de la digestibilité (tableau 3 a)

a) A partir des paramètres fécaux (seuls ou associés)

MATF permet de prévoir la dMO avec une précision du même ordre que celle observée sur les différents fourrages du tableau 1. La précision obtenue avec MSEF 
Tableau 3 a

Précision de la prévision de la digestibilité de la matière organique (p. 100) à partir des caractéristiques des fèces, des fèces et des fourrages offerts, des fourrages offerts.

Accuracy of organic matter digestibility prediction from faeces alone, faeces + herbage and herbage characteristics.

\begin{tabular}{|c|c|c|c|c|c|c|}
\hline \multirow{3}{*}{$\begin{array}{l}\text { Variables } \\
\text { Variable }\end{array}$} & \multicolumn{4}{|c|}{$\begin{array}{l}\text { Ray-grass d'Italie } \\
\text { Italian ryegrass }\end{array}$} & \multicolumn{2}{|c|}{$\begin{array}{l}\text { Dactyle } \\
\text { Cocksfoot }\end{array}$} \\
\hline & \multicolumn{2}{|c|}{$\begin{array}{l}\mathbf{1}^{\text {er }} \text { cycle } \\
\text { (19) }\end{array}$} & \multicolumn{2}{|c|}{$\begin{array}{l}2^{\circ} \text { cycle } \\
(10)\end{array}$} & \multicolumn{2}{|c|}{$\begin{array}{c}1^{\text {er }}+3^{\circ} \text { cycle } \\
(17)\end{array}$} \\
\hline & $\mathrm{R}$ & Syx & $\mathbf{R}$ & Syx & $\mathbf{R}$ & Syx \\
\hline \multicolumn{7}{|l|}{$\begin{array}{l}\text { a) Fèces } \\
\text { Faeces }\end{array}$} \\
\hline MATF & 0,941 & 2,42 & 0,933 & 2,09 & 0,856 & 1,99 \\
\hline MSEF & 0,893 & 3,22 & 0,871 & 2,84 & 0,544 & 3,22 \\
\hline DENSF & 0,954 & 2,06 & 0,849 & 3,05 & 0,745 & 2,57 \\
\hline $\mathrm{CBF} \ldots \ldots \ldots$ & 0,913 & 2,92 & 0,845 & 1,89 & 0,681 & 2,82 \\
\hline DENSF, MSEF & 0,963 & 1,88 & 0,847 & 3,23 & 0,739 & 2,60 \\
\hline$\ldots \ldots \ldots \ldots \ldots \ldots$ & 0,937 & 2,46 & 0,916 & 2,45 & 0,937 & 2,46 \\
\hline MATF, DENSF $\ldots \ldots \ldots \ldots \ldots \ldots$ & 0,962 & 2,02 & 0,941 & 2,10 & 0,855 & 2,07 \\
\hline $\begin{array}{l}\text { MATF, CBF } \ldots \ldots \ldots \ldots \ldots \ldots \ldots \\
\text { MATF, CBF, NDFF, ADFF, DENSF, }\end{array}$ & 0,946 & 2,46 & 0,946 & 2,01 & 0,858 & 2,05 \\
\hline MSEF $\ldots \ldots \ldots \ldots \ldots \ldots \ldots$ & 0,989 & 1,28 & 0,982 & 1,80 & 0,935 & 1,67 \\
\hline \multicolumn{7}{|l|}{$\begin{array}{l}\text { b) Fèces }+ \text { fourrages proposés } \\
\text { Faeces + herbage }\end{array}$} \\
\hline MATF, MATP $\ldots$. & 0,942 & 2,47 & 0,944 & 2,03 & 0,903 & 1,71 \\
\hline MATF, СВF, MATP $\ldots \ldots \ldots$ & 0,946 & 2,46 & 0,954 & 2,00 & 0,903 & 1,78 \\
\hline MATF, CBF, МАTP, CBP $\ldots \ldots \ldots$ & 0,948 & 2,51 & 0,960 & 2,05 & 0,914 & 1,75 \\
\hline $\begin{array}{l}\text { MATF, CBF, DENSF, MSEF, ADFF, } \\
\text { NDFF, MATP, CBP } \ldots \ldots \ldots \ldots\end{array}$ & 0,989 & 1,36 & 0,987 & $(2,58)$ & 0,951 & 1,64 \\
\hline $\begin{array}{l}\text { c) Fourrages proposés } \\
\text { Herbage }\end{array}$ & & & & & & \\
\hline MATP $\ldots$. & 0,903 & 3,07 & 0,634 & 4,43 & 0,935 & 2,95 \\
\hline MATP, СВР & 0,938 & 2,56 & 0,797 & 3,73 & 0,858 & 1,74 \\
\hline
\end{tabular}

MATF Matières azotées fécales (\% MO) - Faecal crude protein content.

MSEF Matières fécales solubles dans l'eau (\% MO) - Faecal water soluble matter.

DENSF Densité des fèces - Specific weight of faeces.

CBF Cellulose brute fécale (\% MO) - Faecal crude fibre.

NDFF Constituants membranaires totaux des fèces (\% MS) - Cell wall constituents.

ADFF Lignocellulose des fèces (\% MS) - Lignocellulose of faeces.

MATP Matières azotées du fourrage offert (\% MO) - Crude protein of offered herbage.

CBP Cellulose brute du fourrage offert \% MO) - Crude fibre of offered herbage. 
est moins bonne qu'avec MATF. La DENSF est plus précise que MSEF sauf dans un cas et est même plus précise que MATF pour le $1^{\text {er }}$ cycle de ray-grass d'Italie. CBF est un paramètre moins intéressant que MATF (sauf dans le cas de $2^{\circ}$ cycle de ray-grass d'Italie). L'association MATF d'une variable explicative pariétale (CBF), non pariétale (MSEF), ou physique (DENSF) n'améliore pas, ou de façon non significative, la précision permise par MATF seule, à l'exception toutefois de DENSF dans le cas du $1^{\text {er }}$ cycle de ray-grass d'Italie.

b) A partir des paramètres fécaux et de ceux des fourrages offerts

MATP ajoutée à MATF et CBF n'augmente la précision que dans le cas du dactyle. L'association de MATP et de CBP à l'ensemble des paramètres fécaux n'améliore pas, ou de manière non significative (cas du dactyle), la précision par rapport aux paramètres fécaux seuls.

c) A partir des teneurs en matières azotées totales et en cellulose brute du fourrage offert

MATP est un critère de prédiction moins satisfaisant que MATF. L'association CBP, MATP permet, sauf dans le cas du dactyle, de prévoir la digestibilité avec une précision plus élevée que MATP mais moins élevée que MATF, ces deux critères étant considérés seuls.

En définitive, c'est l'ensemble des paramètres fécaux qui permet de prévoir la dMO avec la meilleure précision.

2. Prévision de la quantité de matière organique digestible ingérée (tabl. $3 \mathrm{~b}$ )

a) A partir des paramètres fécaux (seuls ou associés)

MATF permet déjà de prévoir la quantité de MOD ingérée avec une précision intéressante $\left(2,5\right.$ à $\left.3,7 \mathrm{~g} / \mathrm{kg} \mathrm{P}^{0,75}\right)$ mais c'est avec l'ensemble des paramètres fécaux, pariétaux et non pariétaux, que cette dernière est la meilleure (tabl. $3 \mathrm{~b}$ ).

b) A partir des paramètres fécaux et de ceux du fourrage offert

Le fait d'ajouter MATP et CBP à MATF permet d'augmenter la précision de la prévision de MOD ingéré par rapport à MATF mais de manière moins nette que lorsqu'on ajoute MATP et CBP à MATF et CBF, particulièrement dans le cas du $2^{\circ}$ cycle de ray-grass d'Italie $(2,33)$ à l'exception toutefois des dactyles. C'est l'adjonction de MATP et CBP à l'ensemble des paramètres fécaux qui conduit à la meilleure réduction de l'erreur de prévision. Toutefois dans le cas du $2^{\mathbf{e}}$ cycle de ray-grass d'Italie, Syx reste élevé malgré un coefficient de corrélation élevé sans doute parce que la prise en compte d'un aussi grand nombre de variables explicatives (8) pour une population de 10 échantillons réduit le nombre de degrés de liberté.

c) A partir des teneurs en matières azotées totales et en cellulose brute du fourrage offert

MATP ou CBP n'augmentent pas la précision de la prévision par rapport à MATF. 
TABLEAU $3 \mathrm{~b}$

Précision de la prévision de la matière organique digestible ingérée $\left(g / \mathrm{kg} P^{\theta, 75}\right)$ à partir des caractéristiques des fèces, des fèces et des fourrages offerts, des fourrages offerts.

Accuracy of the prediction of digestible organic matter intake $\left(\mathrm{g} / \mathrm{kg} P \mathrm{~W}^{0.75}\right)$ from faecal, faecal + forage and forage characteristics.

\begin{tabular}{|c|c|c|c|c|c|c|}
\hline \multirow{3}{*}{$\begin{array}{l}\text { Variable } \\
\text { Variable }\end{array}$} & \multicolumn{4}{|c|}{$\begin{array}{l}\text { Ray-grass d'Italie } \\
\text { italian ryegrass }\end{array}$} & \multirow{2}{*}{\multicolumn{2}{|c|}{$\begin{array}{c}\text { Dactyle } \\
\text { Cock's foot } \\
1^{\omega 2} \text { et } 3^{\circ} \text { cyclo } \\
(17)\end{array}$}} \\
\hline & \multicolumn{2}{|c|}{$\begin{array}{c}1^{\text {ar }} \text { cycle } \\
(19)\end{array}$} & \multicolumn{2}{|c|}{$\begin{array}{c}2^{2} \text { cycle } \\
(10)\end{array}$} & & \\
\hline & $\mathrm{R}$ & $5 y x$ & $\mathbf{R}$ & Syx & $\mathbf{R}$ & Syx \\
\hline \multicolumn{7}{|l|}{$\begin{array}{l}\text { a) Fèces } \\
\text { Faeces }\end{array}$} \\
\hline $\begin{array}{l}\text { MATF } \ldots \ldots \ldots \ldots \ldots \\
\text { CBF } \ldots \ldots \ldots \ldots \ldots \ldots \ldots \ldots \\
\text { MATF, CBF } \ldots \ldots \ldots \ldots \ldots \\
\text { MATF, CBF, DENSF, MSEF, NDFF, } \\
\text { ADFF }\end{array}$ & $\begin{array}{l}0,927 \\
0,907 \\
0,931\end{array}$ & $\begin{array}{l}3,69 \\
4,15 \\
3,69\end{array}$ & $\begin{array}{l}0,902 \\
0,917 \\
0,917\end{array}$ & $\begin{array}{l}2,54 \\
2,35 \\
2,51\end{array}$ & $\begin{array}{l}0,840 \\
0,671 \\
0,842\end{array}$ & $\begin{array}{l}3,43 \\
4,68 \\
3,53 \\
2,35\end{array}$ \\
\hline \multicolumn{7}{|l|}{$\begin{array}{l}\text { b) Fèces + fourrages offerts } \\
\text { Faeces + herbage }\end{array}$} \\
\hline 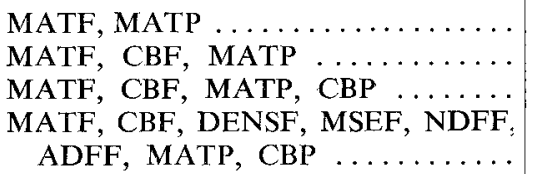 & $\begin{array}{l}0.928 \\
0.933 \\
0.947 \\
0.988\end{array}$ & $\begin{array}{l}3,78 \\
3,76 \\
3,49 \\
2,01\end{array}$ & $\begin{array}{l}0.907 \\
0.919 \\
0.950 \\
0.906\end{array}$ & $\begin{array}{l}2,65 \\
2,63 \\
2,33 \\
4,35\end{array}$ & $\begin{array}{l}0,849 \\
0,852 \\
0,861 \\
0,989\end{array}$ & $\begin{array}{l}3,46 \\
3,56 \\
3,59 \\
1,28\end{array}$ \\
\hline $\begin{array}{l}\text { c) Fourrages offerts } \\
\text { Herbage }\end{array}$ & & & & & & \\
\hline $\begin{array}{l}\text { MATP } \quad \ldots \ldots \ldots \ldots \ldots \ldots \ldots \ldots \\
\text { MATP, }\end{array}$ & $\begin{array}{l}0,896 \\
0,928\end{array}$ & $\begin{array}{l}4,36 \\
3,78\end{array}$ & $\begin{array}{l}0,650 \\
0.907\end{array}$ & $\begin{array}{l}447 \\
2,65\end{array}$ & $\begin{array}{l}0,749 \\
0,849\end{array}$ & $\begin{array}{l}4,18 \\
3,46\end{array}$ \\
\hline $\begin{array}{l}\text { MOD ingérée moyenne }(\bar{x}) \ldots \\
\text { Mean DOM intake } \ldots \ldots \ldots \ldots\end{array}$ & \multicolumn{2}{|c|}{$43,04 \pm 9,6$} & \multicolumn{2}{|c|}{$37,27 \pm 5,5$} & \multicolumn{2}{|c|}{$39,61 \pm 6,1$} \\
\hline $\begin{array}{l}\text { Meilleure précision de l'estimation } \\
\text { exprimée en p. } 100 \text { des quantités } \\
\text { moyennes de MOD ingérées (CV \%) } \\
\text { Better accuracy of the estimation ex- } \\
\text { pressed as \% of mean DOM intakes, }\end{array}$ & \multicolumn{2}{|c|}{4,7} & \multicolumn{2}{|c|}{6,3} & \multicolumn{2}{|c|}{3,2} \\
\hline
\end{tabular}

Cf. légende tableau 3 a - See table 3 a. 
En définitive, la prévision de la quantité de MOD ingérée est la meilleure avec :

- la teneur en MAT et $\mathrm{CB}$ des fèces et du fourrage offert dans le cas de $2^{\circ}$ cycle de ray-grass d'Italie $\left(2,33 \mathrm{~g} / \mathrm{kg} \quad \mathrm{P}^{0,75}\right.$ soit $6,3 \mathrm{p} .100$ la MOD ingérée moyenne),

- l'ensemble des paramètres fécaux, MATP et CB, dans les detux autres cas $\left(2,01\right.$ et $1,28 \mathrm{~g} / \mathrm{kg} \mathrm{P}^{0,75}$ soit, respectivement 4,7 et 3,2 p. 100 de la MOD ingérée moyenne).

Cette précision de la prévision est donc très intéressante.

\section{Utilisation des équations dans la pratique}

1. Influence du niveau d'alinentation sur la digestibilité et la teneur en matières azotées des fèces

A partir des résultats d'un essai effectué antérieurement par Demarouilly (résultats non publiés), nous avons vérifié si la dMO et les MATF dépendaient du niveau d'alimentation : un ray-grass d'Italie a été distribué à des moutons à volonté ou en quantité limitée, les quantités de matière sèche ingérées étant respectivement de 73,0 et de $43,9 \mathrm{~g} / \mathrm{kg} \mathrm{P}^{0,75}$. La digestibilité a été respectivement de 78,8 et de 80,8 p. 100 et les teneurs en matières azotées fécales de 20,3 et 20,9 (différences non significatives dans les deux cas).

\section{Cas de l'herbe complémentée par des concentrés}

Nous avons exploité les résultats de deux essais de mesure de digestibilité sur des moutons à l'auge (Chenost, Grenet \& Demarourtly, 1985) qui recevaient d'une part, de l'herbe à volonté $\left(1^{\mathrm{er}}, 2^{\prime \prime}\right.$ et $3^{\prime \prime}$ cycles de ray-grass d'Italie, de ray-grass anglais et de fétuque des prés) et, d'autre part, la même herbe complémentée soit par de l'orge ( 21 à $25 \mathrm{p}$. 100 de la ration), soit par un aliment concentré composé de 75 p. 100 de maïs, 22 p. 100 de tourteau de soja et 3 p. 100 de minéraux (33 à 41,6 p. 100 de la ration).

Ces essais ont montré que la digestibilité de la ration totale présentait une liaison avec la digestibilité de l'herbe et la proportion de concentré dans la ration. Cette liaison devenait plus étroite lorsque la digestibilité de l'herbe diminuait et, cela, d'autant plus que la proportion de concentré augmentait. La teneur en MAT de la ration a été en moyenne de 18,2 p. 100, elle n'était pas ou peu affectée par la présence de concentré; il est vrai que MATP seule était déjà élevée (16,8 p. 100 en moyenne).

La relation entre dMO et MATF avec l'herbe seule ne diffère pas significativement de celle obtenue avec l'herbe complémentée. La précision de la prévision de la dMO de la ration totale obtenue pour l'ensemble des cycles, après élimination de 3 fourrages de faible digestibilité, est bonne $(S y x= \pm 1,3 ; n=24)$. Elle est un peu inférieure à celles obtenues avec les $1^{\text {pr }}$ et $2^{\prime \prime}$ cycles d'une part (Syx $=1,56$; $\mathrm{n}=14$ ) et avec les $3^{\text {e }}$ cycles d'autre part $(\operatorname{Syx}=0,75 ; \mathrm{n}=10)$. 


\section{Utilisation des équations pour bovins}

Nous avons conduit par ailleurs (CHENOST, à paraître) un essai destiné à étudier les différences dans la dMO et la quantité de MOD ingérée entre des moutons d'une part et des vaches en lactation et des taurillons d'autre part. Les fourrages (fig. 3) avaient été offerts, à volonté, en vert, à l'auge à chaque catégorie d'animaux. Pour un fourrage donné, les dMO et MATF sont plus importantes chez le bovin que chez le mouton. Les droites de régression $\mathrm{dMO}=\mathrm{f}$ (MATF) ont le même coefficient de régression (1,43 et 1,41 respectivement pour les moutons et les bovins), elles sont donc presque parallèles. Elles diffèrent par leurs ordonnées à l'origine, celle des bovins étant supérieures de 2 points.

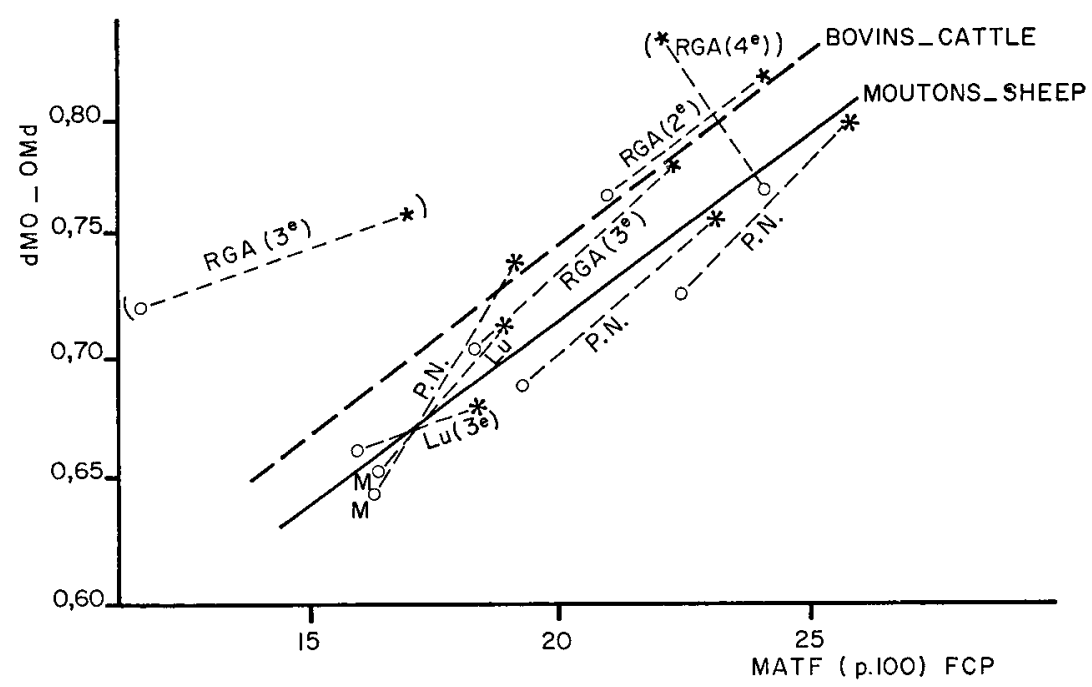

FIG. 3

Comparaisons sur 7 mesures (1) de digestibilité,

des relations $d M O=f(M A T F)$ obtenues sur moutons $\left({ }^{*}\right)$ et sur bovins $(0)$.

Comparison with 7 digestibility trials (1) of $O M d=f(F C P)$ relationships obtained with sheep and cattle.

Moutons (sheep) : $\mathrm{dMO}=42,6+1,43 \mathrm{MATF} \pm 2,62 ; \mathrm{r}=0,840 ; \mathrm{n}=7$.

Bovins (cattle) : $\mathrm{d} M O=44,6+1,41 \mathrm{MATF} \pm 2,36 ; \mathrm{r}=0,881 ; \mathrm{n}=7$.

(1) Nous avons éliminé un échantillon de RGA $3^{\text {e }}$ cycle et un échantillon RGA $4^{\text {e }}$ cycle pour lesquels les MATF ne rentrent pas, à dMO donnée, dans la population (entre parenthèses sur le graph:que), très vraisemblablement en raison de transitions trop courtes entre périodes de mesures dues au manque d'heibe durant l'essai (été 1980).

One sample of Italian ryegrass (3rd cycle) and one sample of perennial ryegrass have been rejected as their FCP content did not enter, at a given OMd, in the population (between brackets in the graph) probably because of too short intervals between 2 OMd measurements (shortage of heibage during summer 1980). 


\section{Discussion}

Les équations basées sur la teneur en matières azotées de la matière organique fécale permettent de prévoir la digestibilité de l'herbe avec une précision tout à fait correcte. Ceci rejoint les conclusions d'ARnold \& Dudzinski (1967) qui considèrent que l'azote fécal est un "prédicteur» de la digestibilité plus intéressant que la digestibilité in vitro des bols cesophagiens. Toutefois, les relations entre dMO et MATF présentent des variations. En outre, leur utilisation dans la pratique suppose certaines précautions.

\section{A. Variation de la relation entre la digestibilité de l'herbe et la teneur en azote des fèces}

On sait (JARRIGE, 1965), que la teneur de chacun des constituants pariétaux dans les fèces dépend, globalement, de la teneur de la fraction indigestible du constituant dans les fourrages. Cette liaison devient encore plus étroite et commune à tous les échantillons si on exprime cette dernière variable en p. 100 de la matière organique sans les glucides solubles, lesquels ne sont pas génératcurs d'indigestible. C'est pourquoi la teneur en constituants pariétaux des fèces est étroitement liée à la teneur en constituants pariétaux indigestibles du fourrage (hémicelluloses indigestibles + cellulose indigestible + lignine), eux-mêmes facteurs déterminant la digestibilité du fourrage. Sur le plan pratique, ces constituants sont longs et coûteux à analyser.

La teneur en constituants cellulaires des fèces varie ainsi avant tout de façon passive, en sens inverse de la teneur des constituants pariétaux. C'est pourquoi la teneur en azote (constituants pour la plus grande part d'origine non pariétale) des fèces est étroitement liée, de façon indirecte (et par un effet de dilution d'une quantité d'azote donnée dans une quantité plus ou moins importante de matière organique non digestible d'origine essentiellement pariétale), à la teneur en constituants pariétaux indigestibles du fourrage, donc à la digestibilité de ce dernier. L'analyse de l'azote est classique, rapide et peu coûteuse.

Cependant, cette liaison présente certaines spécificités. En effet la teneur en azote dans les fèces correspondant aux repousses feuillues, surtout d'automne, est plus élevée que dans les fèces correspondant aux premiers cycles de digestibilité équivalente. Il est encore difficile de dire si cette augmentation est due à une diminution de la digestibilité vraie de l'azote du fourrage ou à une augmentation de l'excrétion de l'azote endogène. La teneur des fèces en azote, bon critère de prédiction de la dMO des fourrages, ne peut donc pas être utilisée pour l'ensemble des différents cycles ou des différentes espèces.

Les variations de la teneur en azote des fèces reflètent déjà, indirectement, celle des constituants pariétaux. C'est probablement pour cette raison que l'adjonction de CBF à MATF, sur l'ensemble de nos échantillons, n'a pas permis d'améliorer la précision de la prévision de la digestibilité. Cette prévision a cependant pu être améliorée sur les échantillons de ray-grass d'Italie et de dactyle (tabl. 3 a) en ajoutant à MATF l'ensemble des paramètres pariétaux, un paramètre non pariétal 
(MSEF) et une caractéristique physique des fèces (DENSF). L'amélioration globale obtenue avec ces critères dépend toutefois de l'espèce végétale et du numéro du cycle. Il en est de même des "étapes" de cette amélioration : dans le cas du raygrass d'Italie l'erreur de prévision diminue déjà avec DENSF et MSEF ( ${ }^{\text {er }}$ cycle) et avec CBF (2'cycle). En revanche ce n'est que l'adjonction simultanée de l'ensemble des différents critères qui permet de réduire l'erreur de prévision (Syx) dans le cas du dactyle.

En définitive, l'azote fécal est un bon critère de prédiction de la digestibilité mais il doit être utilisé avec certaines précautions. L'addition d'autres paramètres chimiques, physico-chimiques ou physiques soit des fèces, soit de l'herbe proposée, augmente la précision de la prévision de la digestibilité. Ceci confirme les résultats antérieurs de JARRIGE (1965) somme des MATF et des matières fécales solubles dans l'eau) et les résultats récents d'Holloway, Estell \& Butts (1981). Ces derniers, à l'aide de corrélations multiples incluant l'indigestible alimentaire et les fractions endogènes fécales, arrivent à prévoir la digestibilité avec une précision relativement correcte mais, surtout, la quantité de matière sèche digestible ingérée par des bœufs en croissance à $0,41 \mathrm{~kg}$ près $(\mathrm{R}=0,860 ; \mathrm{n}=39)$.

Il conviendrait de poursuivre dans cette voie en insistant plus particulièrement sur la meilleure connaissance des causes de variation des différentes fractions fécales qui sont d'origine alimentaire, microbienne et endogène (JARrige, Journet \& VerRITE, 1978).

On a vu que la relation entre dMO et MATF dépendait, entre autres, de l'espèce végétale. La prévision de la digestibilité des prairies naturelles, de composition botanique complexe, sera par conséquent plus délicate que dans le cas des prairies monospécifiques.

Enfin, si la quantité de matière organique digestible ingérée des fourrages étudiés peut être prévue avec une bonne précision $\left(1,28\right.$ à $\left.2,33 \mathrm{~g} / \mathrm{kg} \mathbf{P}^{0,75}\right)$ cela tient certainement au fait que nous avons ajouté, aux paramètres fécaux, deux paramètres de la composition du fourrage (MATP et CBP), eux-mêmes assez bons prédicteurs de quantités ingérées et, aussi, de la digestibilité.

\section{B. Utilisation de ces relations}

\section{Influence du niveau d'alimentation sur dMO et MATF et les relations dMO/MATF}

On sait que le niveau d'alimentation a une influence directe sur la digestibilité des fourrages. Toutefois la digestibilité des fourrages verts diminue peu lorsque le niveau d'alimentation augmente (ANDERSEn et al., 1959 et Raymond, Harris \& Kemp, 1955). On peut done penser que la relation dMO/MATF est peu modifiée.

Toutefois, dans le cas d'une alimentation à volonté, la quantité de MAT excrétée et les quantités de fèces émises sont plus importantes que dans le cas d'une alimentation limitée. Or MATF est le rapport, en p. 100, de la quantité de MAT à la quantité de matière organique fécale. Comme les deux membres de ce rapport augmentent simultanément, sa valeur reste la même et, par là, la relation dMO/MATF est peu affectée. 
Un essai conduit antérieurement par Demaroullly (1963 et 1966) sur vaches laitières montre qu'il doit être possible, sans commettre une grande erreur, d'appliquer les courbes obtenues avec des animaux alimentés à volonté à des animaux alimentés en quantité limitée.

C'est aussi ce que montrent Hutton \& Jury (1964) qui n'observent pas de différence dans les rapports ingéré/fèces («intake factor») entre des vaches taries recevant de l'herbe en affouragement en vert, soit en quantité limitée au niveau de l'entretien, soit à volonté.

On peut penser, en outre, que la pression de pâturage généralement adoptée pour les animaux à l'herbe se rapproche assez bien des conditions d'alimentation à volonté, ce qui contribuerait à diminuer l'erreur éventuelle.

2. Cas de complémentation du fourrage avec des aliments concentrés (à base de céréales)

La complémentation en aliment concentré peut modifier simultanément la digestibilité de l'herbe et la composition des fèces.

Les résultats de Chenost, Grenet \& Demarouilly (1985) permettent de penser que le concentré n'affecte pas la relation entre dMO et MATF obtenue avec de l'herbe seule. Dans le cas du pâturage avec complémentation on pourrait donc prévoir, avec la même équation et avec une précision relativement bonne, la digestibilité de la ration globale et, par là, les quantités respectives d'herbe et de concentré ingérées (ces dernières étant connues par définition) si l'on mesure les quantités totales de fèces émises.

Ces résultats demandent cependant à être confirmés sur un plus grand nombre d'échantillons d'espèces botaniques et de cycles différents et avec des complémentations plus variées en quantité et en nature. Ils doivent en effet dépendre, d'une part de la différence de digestibilité entre le fourrage et le concentré, d'autre part du numéro du cycle de repousses de l'herbe (en effet dans l'essai mentionné plus haut, les liaisons entre la digestibilité de la ration totale et $\mathrm{MATF}_{\emptyset}$ étaient plus étroites pour les $1^{\text {er }}$ et $2^{\circ}$ cycles que pour le $3^{\circ}$ ).

\section{Utilisation de ces équations pour les bovins}

On sait que l'aptitude digestive des bovins est supérieure à celle des moutons (Blaxter, Waiman \& Davidson, 1966 ; Playne, 1978 ; Carle \& Dulphy, non publié ; Chenost \& MARTIn-Rosset, à paraître). La comparaison des liaisons dMO/ MATF entre moutons et bovins a fait l'objet de travaux limités qui conduisent à des réponses divergentes selon les auteurs : 


\begin{tabular}{|c|c|}
\hline Auteurs & $\begin{array}{l}\text { Conclusions, suivant les auteurs, sur les différences } \\
\text { dans les relations dMO/MATF entre ovins et bovins }\end{array}$ \\
\hline $\begin{array}{l}\text { SANSON \& LANGLANDS } \\
\quad \text { (LANGLANDS, 1975) }\end{array}$ & $\begin{array}{l}\text { Coefficient de régression non différents } \\
\text { Ordonnées à l'origine }:+11,2 \text { pour les ovins }\end{array}$ \\
\hline Corbett $(1960)$ & $\begin{array}{l}\text { MATF vaches laitières > MATF taurillons ou génisses } \\
\text { (l'utilisation d'une même équation conduit à une erreur dans } \\
\text { la prévision de dMO) }\end{array}$ \\
\hline MINSON \& RAYMOND (1958) & $\begin{array}{l}\text { « Erreur d'application } » \text { lorsqu'on transpose aux bovins la } \\
\text { courbe déterminée sur ovins }\end{array}$ \\
\hline LANGLANDS (1975) & $\begin{array}{l}\text { dMO = } \mathbf{f}(\mathrm{MATF}) \text { non différentes entre bovins et ovins } \\
\text { (relation insuffisamment précise pour différencier l'aptitude } \\
\text { au tri d'espèces animales différentes au pâturage) }\end{array}$ \\
\hline Chenost (présente étude) & $\begin{array}{l}\text { Coefficients de régression non différents } \\
\text { Ordonnées à l'origine }:+2 \text { points pour les bovins }\end{array}$ \\
\hline
\end{tabular}

Dans notre essai, il aurait fallu disposer d'un plus grand nombre de mesures pour définir un coefficient de correction plus précis, non seulement entre les deux espèces animales mais également, pour les bovins, entre le sexe, l'âge et le stage physiologique.

Pour le moment on pourra, sans commettre une trop grande erreur, prévoir la digestibilité chez le bovin à partir d'une teneur MATF bovin donnée en ajoutant l'écart de digestibilité compris entre les deux courbes de la figure 3, soit environ 2 points de digestibilité pour une MATF variant de 10 à 25 p. 100 . On pourra surtout classer de façon relative la digestibilité de l'herbe ingérée par des bovins au pâturage à partir de la teneur en MAT des fèces correspondantes qu'ils auront émises.

En définitive, bien qu’il soit théoriquement nécessaire d'effectuer pour chaque essai au pâturage des mesures de digestibilité sur animaux en cage recevant la même herbe à l'auge que les animaux au pâturage (Schneider \& FlatT, 1975; conclusions du 4" Groupe Européen sur le Pâturage, Theix, 1981) l'ensemble des équations proposées ici peut déjà aider les expérimentateurs à apprécicr la digestibilité de l'herbe pâturée dans le cas des prairies monospécifiques et, surtout, à classer les digestibilités des différentes prairies, et ceci, aux différents cycles d'exploitation de l'herbe.

Des prélèvements de fèces, au sol ou dans le rectum, permettront de constituer un échantillon représentatif des fèces émises pendant la présence des animaux sur la prairie. La fréquence des prélèvements dépendra du système de pâturage adopté. Elle devra être telle que l'indigestible récolté corresponde à l'herbe ingérée.

Les équations établies pour les deux prairies naturelles étudiées (prairie Normande, prairie de demi-montagne d'Auvergne) pourront être utilisées de la même façon. En revanche, l'utilisation de ces équations pour d'autres prairies plurispécifiques est à déconseiller. En effet, la composition botanique du cotvert végétal des prairies naturelles est extrêmement variable. Or, on a vu que la relation entre la 
dMO et l'azote fécal dépend, entre autres, de l'espèce botanique. Il conviendra donc dans ce cas d'être très prudent et, à la limite, soit de procéder parallèlement à des mesures de digestibilité sur des animaux recevant de l'herbe fauchée à l'auge, soit, si cela esí impossible, de recourir à d'autres techniques d'estimation de la digestibilité (attaque à la pepsine-cellulase ou digestibilité in vitro d'échantillons représentatifs de l'herbe présente ou d'échantillons de bols cesophagiens).

Reçu en juin 1984. Accepté en avril 1985.

\section{Remerciements}

Nous tenons à remercier $\mathbf{M}^{\mathrm{me}}$ Brigitte Michalet-Doreau, Ingénieur au Laboratoire, pour l'aide précieuse qu'elle nous a apportée dans le calcul des corrélations (exploitation du programme « Seebeck» sur ordinateur).

\section{Summary}

\section{Use of faecal nitrogen and of some faecal parameiers for estimating herbage intake in grazing animals}

This study was made to define equations for predicting digestibility of grazed herbage from faecal criteria. The conditions of application of these equations in practice are discussed.

To that end, the digestibility of 1230 samples of the 10 main meadowplant varietics cultivated in France and of two natural pastures, was measured in sheep.

Regression equations were calculated in relating organic matter digestibility (OMd) of green forages to crude protein and crude fibre contents (FCP and FCF; expressed as percent of organic matter) determined on the corresponding faeces. Forage digestibility ranged from 55 to 80 p. 100 and $\mathrm{FCP}$ content from 8 to $30 \mathrm{p} .100$.

Relationships between OMd and FCP were close and FCF added to FCP did not significantly improve the accuracy of MOd prediction.

The equations and the corresponding curves ( 26 of the 2 nd degree, 5 of the 1st degree) are therefore presented only as a function of FCP. These 31 equations were established for each variety and each cycle. It was observed that for the same FCP and according to the successive cycles :

- digestibility decreased whatever the botanical variety;

- differences of digestibility betwecn varieties increased; same.

- classification of the different varieties according to their digestibility was not the

The coefficients of correlation ranged between 0.887 and 0.958 for the first cycles and between 0.735 and 0.910 for the different regrowths. The accuracy of OMd prediction (Syx) was, on an average, 2.2. to 2.5 points ( 3 values out of 31 exceeded 3 points). It was close and sometimes even better than that obtained from the crude protein and crude fibre content of the forage offered (PCP and PCF, respectively).

Using a restricted number of samples (29 Italian ryegrass, 17 cocks foot), we tried to improve the prediction accuracy (Syx $=1.28$ and 1.67 points respectively for Italian ryegrass and cock's foot) by adding to FCP some chemical faecal criteria : FCF, soluble dry matter FWDM), cell wall components (CWCF) and lignocellulose van SoesT (FAD) as well as physical faecal criteria : faeces density (FD). It was also possible to estimate the digestible organic matter intake with accuracy $(1.28$ to $2.33 \mathrm{~g} / \mathrm{kg} \mathrm{W0.75})$ by adding PCP and PCF to all these faecal criteria. 
The conditions of application of these equations in practice are discussed : for predicting digestibility of grazed herbage by cattle, with or without grazing supplementation and for levels of feeding close to ad libitum.

These equations established for monospecific pastures may be used in practice under certain conditions described in this paper. Using a sample of faeces representative of a given grazing period, these equations allow to predict and classify the digestibilities of herbage grazed by sheep and cattle.

The problem of natural plurispecific pastures has not been solved. It would be necessary either to use digestibility measurements in sheep receiving in parallel grass cut from the same pasture or laboratory measurements (cellulase, nylon bags, DIV) allowing the prediction of OMd from a sample of grass representative of the grazed herbage (cutting, oesophageal bolus).

Key words : Relationships herbage digestibility ; faecal characteristics.

\section{Liste des abréviations \\ List of abbreviations}

dMS digestibilité de la matière sèche (p. 100)

DMd dry matter digestibility (\%)

dMO digestibilité de la matière organique (p. 100)

OMd organic matter digestibility (\%)

MODI quantité de matière organique digestible ingérée $\left(\mathrm{g} / \mathrm{kg} \mathrm{P}^{0,75}\right)$

DOMI digestible organic matter intake $\left(\mathrm{g} / \mathrm{kg} \mathrm{W}^{0.75}\right)$

MATF teneur en matières azotées de la matière organique fécale $(\mathrm{g} / \mathrm{kg})$

FCP crude protein content in faecal organic matter $(\mathrm{g} / \mathrm{kg})$

$\mathrm{CBF}$ teneur en cellulose brute de Weende de la matière organique fécale $(\mathrm{g} / \mathrm{kg})$

FCF crude fibre content in faecal organic matter $(\mathrm{g} / \mathrm{kg})$

ADFF lignocellulose Van Soest des fèces (p. 100 MS)

$F A D F \quad$ lignocellulose (Van Soest) in faecal dry matter

NDFF teneur en parois (Van Soest) des fèces (p. $100 \mathrm{MS}$ )

FNDF cell wall content (Van Soest) of faeces (\% DM)

DENSF densité des fèces

FSG specific weight of faeces

MSEF teneur des fèces en matières solubles dans l'eau (p. 100 MS)

FWSM water soluble faecal matter content (\% DM)

MATP teneurs en matières azotées du fourrage proposé $(\mathrm{g} / \mathrm{kg} \mathrm{MO})$

$P C P \quad$ crude protein content of forage offered $(\mathrm{g} / \mathrm{kg} \mathrm{OM})$

CBP teneur en cellulose brute du fourrage proposé $(\mathrm{g} / \mathrm{kg} \mathrm{MO})$

PCF crude fiber content of forage offered $(\mathrm{g} / \mathrm{kg} \mathrm{OM})$

$\mathrm{r} \quad$ coefificient de corrélation du $1^{\text {cr }}$ degré

coefficient of correlation - 1st degree

$\mathrm{R} \quad$ coefricient de corrélation multiple ou du $2^{\circ}$ degré coefficient of correlation - 2nd degree

Syx écart type réduit de la régression

stanilard error of regression 


\section{Références bibliographiques}

Andersen P.E., Reid J.T., Anderson M.J., Stoud J.W., 1959. Influence of level of intake upon the apparent digestibility of forages and mixed diets by ruminants. J. Anim. Sci., 18, 1299-1307

Andrieu J., Weiss Ph., 1981. Prévision de la digestibilité et de la valeur énergétique des fourrages verts de graminées et de légumineuses. In Prévision de la valeur nutritive des aliments des ruminants, I.N.R.A. Paris, 61-79.

ARNold C.W., Dudzinski M.L., 1963. The use of faecal nitrogen as an index for estimating the herbage intake of grazing animal. J. Agric. Sci., 61, 33-40.

ARnold C.W., Dudzinski M.L., 1967. Comparison of faecal nitrogen regressions and in vitro estimates of diets digestibility for estimating the consumption of herbage by grazing animals. J. Agric. Sci., 68, 213-219.

Aufrere J., 1982. Etude de la prévision de la digestibilité des fourrages par une méthode enzymatique. Ann. Zootech., 31, 111-130.

Blaxter K.L., Wainman F.W., Davidson J.L., 1966. The voluntary intake of food by sheep and cattle in relation to their energy requirements for maintenance. Anim. Prod., 8, 75-83.

Chenost M., Grenet E., Demarquiley C., 1985. Influence of supplementation on herbage digestibility and on faeces characteristics with sheep. XVth Internat. Grassld. Congr. (sous presse).

Corbetr J.L., 1960. Faecal index techniques for estimating herbage consumption by grazing animals. Proc. VIIIth Int. Grassl. Congr. Paper $1 \mathrm{~B} / 2,438-442$.

Demarquilly C., 1963. Influence de la nature du pâturage sur la production laitière et la composition du lait. Ann. Zootech., 12, 69-104.

Demarquilly C., 1966. Valeur alimentaire de l'herbe des prairies temporaires aux stades d'exploitation pour le pâturage. II - Quantités ingérées par les vaches laitières. Ann. Zootech., 15, 147-169.

Demarquilly C., Andrieu J., Sauvant D., 1978. Tableaux de la valeur nutritive des aliments. In L'Alimentation des Ruminants, chapitre 17, pp. 519-577. I.N.R.A. Paris.

Greenhalgh J.F.D., CorbetT J.L., 1960. The indirect estimation of the digestibility of pasture herbage. I - Nitrogen and chromogen as faecal index substances. J. Agric. Sci., 55, 371-376.

Greenhalgh J.F.D., Corbett J.L., MC Donald I., 1960. The indirect estimation of the digestibility of pasture herbage. II - Rcgressions of digestibility on faccal nitrogen concentration : their determination in continuous digestibility trials and the effect of various factors on their accuracy. J. Agric. Sci., 8, 377-386.

Greenitalgh J.F.D., Reid G.W., MC Donald I,, 1966. The indirect estimation of the digestibility of pasture herbage. IV - Regressions of digestibility of faecal nitrogen concentration : effects of different fractions of the herbage upon within and between period regressions. J. Agric. Sci., 66, 277-283.

Holechek J.L., Vavra M., Pieper R.D., 1982. Methods for determining the nutritive quality of range ruminant diets : a review. J. Anim. Sci., 54, 363-376.

Holloway J.W., Estell R.E., Butts Jr W.T., 1981. Relationship between faecal compounds and forage consumption and digestibility. J. Anim. Sci., 52, 836-848.

Hutton J.B., JURY K.E., 1964. Studies of the nutritive value of New Zealand dairy pastures. III - The comparative value of various feed-faeces relationships in herbage intake studies with dairy cattle. New Zealand. J. Agric. Res., 7, 583-595.

JARRIGE R., 1965. The composition of sheep faeces and its relation to forage digestibility. Proc. 9th Internat. Grassl. Congress, São-Paulo, 809-814.

Jarrige R., Journet M., Verite R., 1978. Azote, utilisation digestive et métabolique. Chapitre 3, pp. 89-105, in L'Alimentation des Ruminants. I.N.R.A. Paris. 
Kennedy W.K., Carter A.H., Lancaster R.J., 1959. Comparison of faecal pigments and faecal nitrogen as digestibility indicators in grazing cattle studies. New Zealand. J. Agric. Res., 2, 627-638.

LanCaster R.J., 1949. Estimation of digestibility of grazed pasture from faeces nitrogen. Nature., 163, 330-331.

LANGLANDS J.P., 1962. Energy intake and its utilization for maintenance by the intensively grazed ruminant. P/l. D. Thesis, University of Aberdeen.

LANGLands J.P., CORBETT J.L., MC Donald I., 1963. The indirect estimation of the digestibility of pasture herbage. III - Regressions of digestibility on faecal nitrogen concentration : effects of species and individuality of animal and of the method of determining digestibility upon the relationships. J. Agric. Sci., 61, 221-226.

LANGLANDS J.P., 1975. Techniques for estimating nutrient intake and its utilization by the grazing ruminants. In : MC DONALD I.W. et WARNER A.C.I. (Eds). Digestion and metabolism in the ruminant. Proc. IV. Int. Symp. Ruminant Physiol. University of New England Publication Unit, 320-332.

Ledu Y.L.P., Penning P.G., 1982. Animal based techniques for estimating herbage intake. In : LeAver J.D. (Ed.). Herbage intake handbook. The British Grassl. Soc. (Publ.), $37-35$.

Meiss J.A.C., 1981. In : Herbage intake by grazing dairy cows. Institute for Livestock Feeding and Nutrition Research «Hoorn», Lelystad, Wageningen, 28-31.

MinSON D.J., RaYmond W.F.C., 1958. Sources of error in the use of faecal index relationships. Grassl. Res. Inst., Exp. in Progr. 10, Animal Report, 1956-1957, 92-96.

Minson D.J., Kemp C.D., 1961. Studies in the digestibility of herbage. IX - Herbage and faecal nitrogen as indicators of herbage organic matter digestibility. J. Brit. Grassl. Soc., 16, 76-79.

Playne M.J., 1978. Differences between cattle and sheep in their digestion and relative intake of a mature tropical grass hay. Anim. Feed Sci. Technol., 3, 41-49.

Raymond W.F.C., Kemp C.D., Kemp A.W., Harris C.E., 1954. Studies in the digestibility of herbage. IV - The use of faecal collection and chemical analysis in pasture studies (b). Faecal index methods. J. british Grassl. Soc., 9, 69-82.

RaYmond W.F.C., Harris C.E., Kemp C.D., 1955. Studies in the digestibility of herbage. VI - The effect of level of herbage intake on the digestibility of herbage by sheep. J. brit. Grassl. Soc., 10, 19-26.

SCHNEIDER B.H., Flatt W.P., 1975. The evaluation of feeds through digestibility experiments. Publication the University of Georgia Press, Athens 30620, U.S.A.

SeEbeck R.M., 1973. The effect of body weight loss on the composition of Braham cross and Africander cross steers. I - Empty body weight, dressed carcass weight and offal components. J. Agric. Sci., 80, 201-210.

Streeter C.L., 1969. A review of techniques used to estimate the in vivo digestibility of grazed forage. J. Anim. Sci., 29, 757-768.

TIlley J.A.M., TERrY R.A., 1963. A two stage technique for in vitro digestion of forage crops. J. brit. Grassl. Soc., 18, 104-111.

VAN Soest P.J., 1967. Development of a comprehensive system of feed analyses and its applications to forages. J. Anim. Sci., 26, 119-128. 